\title{
Response and place learning in crayfish spatial behavior
}

\author{
A. J. Tierney ${ }^{1} \cdot$ A. Baker ${ }^{1} \cdot$ J. Forward ${ }^{1} \cdot$ C. Slight ${ }^{1} \cdot$ H. Yilma ${ }^{1}$ \\ Published online: 20 August 2018 \\ (C) Psychonomic Society, Inc. 2018
}

\begin{abstract}
Previous studies have demonstrated that animals use both environmental cues and egocentric information when orienting in mazes or nature. These two strategies have been examined separately in some species, yielding information on the specific properties associated with each. We examined spatial learning in crayfish (Orconectes rusticus) using an apparatus that required animals to orient under four different conditions: using egocentric (response) cues alone, response cues with inconsistent external (place) cues present, place cues with inconsistent response cues present, and place cues with consistent response cues present. Results demonstrated that crayfish could successfully learn a maze task using response cues alone and when external visual and tactile cues provided inconsistent information. Animals were markedly less successful at learning the task using place cues while disregarding inconsistent response information. We also found that more animals learned successfully when response and external cues were presented in a redundant format where both cues indicated the correct turn. Finally, we found that some crayfish were able to learn a single reversal when trained using response information alone and when response and external cues were presented in the redundant format. We consider these results in the light of findings from other species, and ideas on learning strategy properties, ecological relevance of strategies, and the possible role of stress coping style in crayfish learning.
\end{abstract}

Keywords Spatial learning $\cdot$ Orientation $\cdot$ Navigation $\cdot$ Egocentric $\cdot$ Crayfish $\cdot$ Crustacean

The ability to orient within a home range is a fundamental and common cognitive process, used daily by many vertebrate and invertebrate species. The strategies that animals use for efficient movement between home and goal locations include both place and response tactics. In the former, animals pay attention to environmental cues, such as landmarks or beacons, whereas in the latter, they remember their own movements, allowing them to follow a precise route to a goal and/or use path integration to return home. In the laboratory, a fruitful approach to understanding place and response spatial learning has involved the use of simple mazes to address how animals locate goals: do they learn about the place where the goal is located using local or distant landmarks, or do they learn about the turns required to reach the goal? The early controversy over which form of learning prevailed was resolved by Restle (1957), who concluded that rats can use both place

A. J. Tierney

atierney@colgate.edu

1 Neuroscience Program, Department of Psychology, Colgate University, Hamilton, NY 13346, USA and response cues to solve spatial tasks, with strategy choice dependent on environmental conditions.

Data from nonrodent species indicate that diverse animals, including fish (Odling-Smee, Boughman, \& Braithwaite, 2008; Rodriguez, Duran, Vargas, Torres, \& Salas, 1994; Salas, Rodríguez, Vargas, Durán, \& Torres, 1996), cuttlefish (Alves, Chichery, Boal, \& Dickel, 2007), and crayfish (Tierney \& Andrews, 2013; Tierney \& Lee, 2011) can learn routes in mazes using both response and intramaze and/or extramaze place cues. In most studies, the maze task was presented in a dual-solution format: the animal could learn a route using response cues, place cues, or both combined, and probe tests determined the chosen strategy. This approach offers valuable information about how animals choose to learn, but does not provide information on the properties of each strategy or how they interact during learning. This information can be acquired by examining each strategy in isolation, as has been done in rats using brain lesions (White, Packard, \& McDonald, 2013) or manipulations of maze cues (Gibson \& Shettleworth, 2005).

In the present experiments, we used the latter approach to examine the ability of crayfish to use a single type of cue, response or place, to solve a maze task. By studying the strategies separately, we aimed to gain insight into each, providing 
comparative information on learning properties that have been well-examined in only a few species. For example, one such property concerns the time course of response and place learning. Packard and McGaugh (1996) discovered that rats use place learning to find a goal in the first week of training, but switch to a response strategy during the second week. In ants, some species initially use path integration to follow routes, but subsequently switch to using beacons or landmarks, allowing for more accurate navigation (Collett, Graham, \& Durier, 2003). Little is known about the time course of route learning in other invertebrates or if it is affected by strategy use.

Another property is the interaction between strategies as animals learn a spatial task. When multiple cues are available, the Rescorla-Wagner model suggests that they compete with each other during learning, with the most salient cue acquiring most associative space. In rats, cue competition is indeed apparent if tasks are staged such that place and response cues conflict (Poldrack \& Packard, 2003; White et al., 2013), but if both cue types lead to the same correct location, place and response learning can occur simultaneously (Packard \& McGaugh, 1996). In making use of both strategies, animals may display enhanced learning relative to their performance in the presence of a single cue (Rodriguez et al., 1994; Schmidt, Jacobson, \& Markus, 2009), a finding referred to in earlier studies as "additivity of cues" (Sutherland \& Mackintosh, 1971). The ability to make simultaneous and cooperative use of multiple spatial cues may be adaptive because, in natural habitats, redundant information can mark the same location that may be crucial for survival. When this is the case, eclectic use of spatial strategies may enable more reliable orientation (Shettleworth, 2005). We hypothesized that, compared with trials using a single cue or two inconsistent cues, crayfish would display enhanced learning in trials where intramaze place cues and turn direction were redundant, leading to the same goal location.

A third property associated with place and response learning is the behavioral flexibility allowed by each strategy. Food locations are often unstable, a situation that should generally favor the ability to learn changes in familiar routes. However, data suggest that learning strategies differ, with place learning associated with greater flexibility than response learning. For example, Kleinknecht et al. (2012) found that reversal learning or strategy switching was displayed in mice that solved a maze task using place cues, but not in mice that used response learning. Likewise, goldfish performed much more accurately in a reversal task when orienting in a maze using spatial rather than response cues (Rodriguez et al., 1994). The generality and significance of this finding would be better understood by examining diverse species, and hence an additional goal of this study was to determine if crayfish were capable of learning a single reversal in our apparatus. Based on limited prior data (Capretta \& Rea, 1967; Costanzo \& Cox, 1971), we hypothesized that they would be and, like other species, would display greater behavioral flexibility when using place compared with response learning protocols.

Large invertebrates such as crustaceans offer excellent models for the study of spatial learning. They possess relatively simple, accessible nervous systems that generate a variety of spatial behaviors including path integration (Zeil, 1998), use of compass directions (Scapini, 2006), and landmark recognition (Cannicci, Barelli, \& Vannini, 2000). For crayfish, studies have shown that they are highly mobile in their natural habitats and able to return to shelters after foraging (Davis \& Huber, 2007; Kamran \& Moore, 2015). In the laboratory, they readily explore new spaces (Drozdz, Viscek, Brudzynski, \& Mercier, 2006; McMahon, Patullo, \& Macmillan, 2005), learn to avoid areas associated with punishment (Bhimani \& Huber, 2016), and remember a maze task for at least 1 week (Tierney \& Andrews, 2013). The present study extends past work by examining how crayfish learn when a cross maze is manipulated to allow reliance on response cues alone, place cues alone, or both cues simultaneously.

\section{Method}

\section{Animals}

Adult $O$. rusticus of both sexes were collected from Payne Creek, Madison Co., New York. Previous studies demonstrated that male and female $O$. rusticus did not differ in their spatial abilities (Tierney \& Andrews, 2013), and hence both sexes were used in the present experiments. All males were in the reproductively active Form I, and females exhibited mature glair glands; all possessed intact antennae, antennules, and appendages. Females ranged in size from $8.0 \mathrm{~g}$ to $17.1 \mathrm{~g}$ (mean $=12.2 \mathrm{~g}$ ) and males ranged in size from $8.8 \mathrm{~g}$ to $17.5 \mathrm{~g}$ (mean $=14.4 \mathrm{~g})$. Prior to being tested, crayfish were held in community tanks $(50 \times 50 \times 25 \mathrm{~cm})$ supplied with constantly flowing dechlorinated tap water at $14^{\circ}-15^{\circ} \mathrm{C}$ under a photoperiod of $12 \mathrm{hr}$ light:12 hr dark. They were fed Purina trout pellets two times per week. Crayfish selected for the experiment were moved to the testing room and placed individually in $10-\mathrm{L}$ tanks at room temperature $\left(20^{\circ}-21^{\circ} \mathrm{C}\right) 5$ days prior to their first exposure to the maze, and they were not fed during this time. The experimental room was on a reverse light cycle with lights on at 19:00 and off at 7:00, and experiments were conducted between 9:00 and 15:00.

\section{Apparatus}

All tests were conducted in a cross-shaped maze constructed entirely from white Plexiglass (see Fig. 1; apparatus was modified from one described previously; Tierney \& Lee, 2011). Arms A and B served as alternate starting locations, 


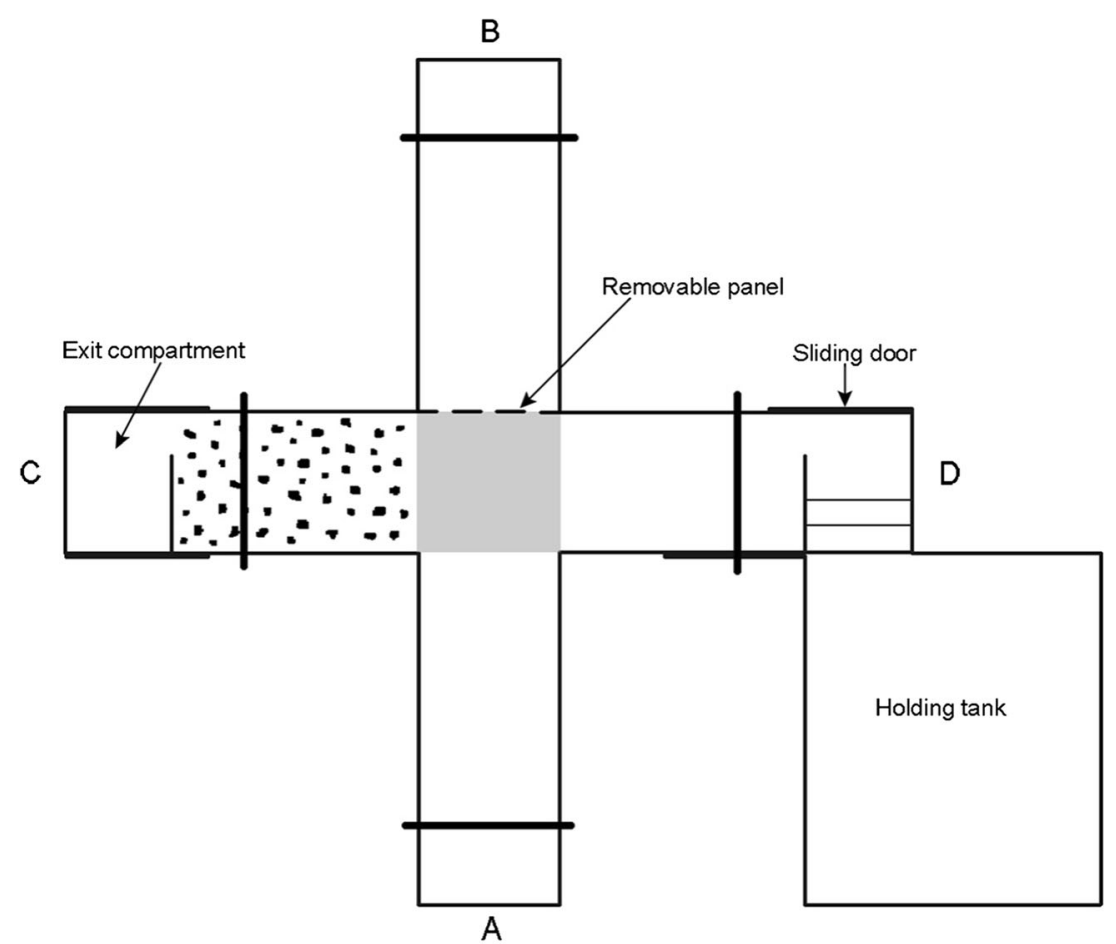

\section{Guillotine doors}

Fig. 1 Maze used to test response and place learning. A panel (removable panel) was used to block one start arm (A or B), creating a T maze. In the configuration shown, arm $\mathrm{C}$ contains the removable rough floor indicated

while arms $\mathrm{C}$ and $\mathrm{D}$ served as goal arms, which each contained two exit windows situated opposite each other and which were equipped with sliding doors such that they could be open or closed. For learning sessions, a removable panel placed at the proximal end of either start arm created a T-maze configuration, and in each trial a crayfish was started at the distal end of the open start arm. Removable panels were located in each goal arm, $6 \mathrm{~cm}$ from the distal end, each with a 6$\mathrm{cm}$ high $\times 3$-cm wide opening through which crayfish walked to reach the end of the arm. The panels created a goal compartment and were situated such that a crayfish in the center of the maze could not see which goal end contained an open exit. The water level in the maze (5-cm deep) came up to the lower edge of the exit window, and removable steps (white Plexiglass blocks forming two 1.5 -cm high steps) were set so that the crayfish could easily climb out of the maze and enter a holding tank. The holding tank $(30-\mathrm{cm}$ long $\times 15-\mathrm{cm}$ wide $\times 10-\mathrm{cm}$ high) was filled with water, and its top was level with the maze exit window. The separation between the maze and the holding tank allowed us to bait the latter with food while ensuring that chemical cues would not influence orientation in the maze.

To eliminate extramaze visual stimuli, the present experiments were conducted with the entire apparatus contained within a six-sided box $(106-\mathrm{cm}$ long $\times 106-\mathrm{cm}$ wide $\times 67$ $\mathrm{cm}$ high) with the interior coated with white paint. A camera by black dots, and arm D contains the stairs, open exit door, and holding tank. Bars across each arm indicate guillotine doors used to confine animals at the beginning and end of each trial

was placed on top of the box with the opening for the lens (3.5 $\mathrm{cm})$ exactly above the center of the maze. The image of the maze was projected to a computer monitor, allowing the experimenter to observe an animal's movements within the maze. Light was provided to the interior of the box by four identical panels containing red, blue, and green LED lights, each panel situated $40 \mathrm{~cm}$ from the camera lens; red, blue, and green lights were independently manipulated from the outside of the box. A door at the front of the box allowed the experimenter to access the maze and place crayfish in a start compartment; when closed, the door formed a wall identical to the other interior walls. A guillotine panel made of clear Plexiglass held the crayfish in a start compartment at the distal end of Arm A or B until the box was closed, at which point it could be manually lifted from outside. Each goal arm (C and D) was also equipped with a guillotine panel, likewise operated from outside the box. These panels were lowered to trap the crayfish and terminate a trial when an animal entered a goal compartment. Experiments were conducted during the animals' dark phase with the room illuminated by light from a single red 13-watt fluorescent bulb and red LED lights within the white box. Animals were transported to the training apparatus under red light, and, once they were situated in the maze, the door to the surrounding white box was closed. The light inside the closed box remained red for $30 \mathrm{~s}$ and then blue and green light was added to the box interior, creating white 
room lighting for each trial and allowing animals to see visual cues in the maze. Prior to reopening the door after a training trial, the lighting was always returned to red only. Crayfish are insensitive to red light (Cronin \& Goldsmith, 1982; McMahon et al., 2005), and hence this protocol reduced the animals' ability to see the location of the door and use the memory of its location as an orientation cue within the maze.

Intramaze place cues included both tactile and visual stimuli. The tactile cue consisted of a floor panel made from clear Plexiglass $(14-\mathrm{cm}$ long $\times 8$-cm wide $\times 0.4-\mathrm{cm}$ thick) and coated with rough pieces of white aquarium gravel (each piece measuring approximately $0.5 \mathrm{~cm}$ in diameter) attached to the Plexiglass with Super Glue. When set in a goal arm, the floor panel extended from the exit compartment panel to the proximal end of the arm. Crayfish had to walk across a smooth or rough surface as they moved down the maze arm, receiving tactile stimuli to mechanoreceptors located on the antennae and walking legs. When walking in the maze, crayfish actively touched their antenna to the walls and floor and probed with walking legs, suggesting that tactile cues were perceived (Tierney \& Lee, 2011). The visual place cue consisted of three strips of black waterproof tape attached to the goal compartment panel, forming two vertical lines from the maze floor to the panel top and one horizontal line $2 \mathrm{~cm}$ from the panel top. Both place cues were always situated together such that the animal could use tactile, visual, or both cue types to identify a maze arm. The opposite arm contained a plain white goal compartment panel and no floor panel, creating the smooth walking surface. All external cues were removable and could be arranged in either goal arm as required by the different learning protocols described below.

\section{General procedures}

On the first experimental day, each crayfish was placed individually in the maze for $1 \mathrm{~h}$ with all arms open. Place cues were situated in either Arm C or D, but all escape doors were closed. Directly following this exploration period, each animal was given two opportunities to escape from the maze. For these practice trials, one door was opened, and the stairs were installed to allow the animal to walk out of the maze to the holding tank baited with two trout food granules (40-50 mg each). The exploration period and practice trials allowed the animals to become familiar with the maze environment and escape task prior to the start of training trials the following day. Animals that did not exit spontaneously after encountering the stairs were confined within the goal compartment and given $10 \mathrm{~min}$ to climb to the holding tank; if they did not do so, they were eliminated from the experiment (two animals). On the second day, training trials began. Each crayfish received a session of 10 training trials/day, and all animals received 7 consecutive days of training. We opted to train animals for 7 days following pilot experiments in which crayfish received up to 14 days of training. We found that if animals did not learn the task within 7 days of testing, additional testing days did not improve performance.

A training trial began when a crayfish was placed in a start compartment and held there by the shut guillotine door. The box surrounding the maze was then closed, and after $30 \mathrm{~s}$ the lighting was adjusted to illuminate the interior. $30 \mathrm{~s}$ after light adjustment, the guillotine door was lifted and the crayfish could walk through the T-maze. During Trial 1 on the first day, the crayfish was allowed to turn either left or right and, if the first turn was to the closed arm, it could retrace its steps and enter the "correct" arm containing the open exit. However, in all subsequent trials the guillotine door was shut as soon as an animal entered an exit compartment, trapping the animal and ending the trial. If the crayfish entered the correct compartment, it was allowed to climb the stairs and exit to the holding tank; if it entered the incorrect compartment, the experimenter immediately opened the box, and transferred the crayfish back to the start compartment. One minute later, the next trial began with the opening of the start compartment door. This procedure reduced the animals' tendency to wander through the maze or develop path habits in which they entered arms multiple times prior to exiting (Tierney \& Lee, 2011).

As a reward for choosing the correct arm and exiting the maze, animals were allowed to spend $3 \mathrm{~min}$ in the holding tank and consume two trout food granules. They were then transferred back to the start compartment to begin another trial. Between trials the water in the maze was thoroughly mixed to disperse any chemical trails the crayfish might have created, and the maze was rinsed and refilled with fresh water between different animals. During each trial, the experimenter watched the animal's movements on the computer monitor outside the box and recorded the turn (to $\mathrm{C}$ or D arm) and the latency to exit the maze. A turn was recorded when the animal's entire body had crossed the line separating the maze center (shaded area in Fig. 1) from an arm. Animals were eliminated from the experiment if the latency to exit the maze exceeded $10 \mathrm{~min}$ in eight or more trials over 2 consecutive days, behavior that appeared to reflect low or no motivation to enter the holding tank. Using this criterion, 22 animals were eliminated during the course of their training.

In Experiment $1(N=56)$, crayfish were randomly assigned to one of four different training protocols (see Fig. 2): (1) Response-learning only (response only). In this group, the crayfish navigated the maze with no tactile or visual cues present. All intramaze external cues were removed such that Arms C and D were identical, each with a smooth floor and a white exit compartment panel. In this and all groups, extramaze visual cues were eliminated by the box that enclosed the maze. Crayfish had to rely solely on an egocentric strategy to learn the location of the exit. To ensure that animals did not use subtle place information unknown to the experimenter, they began five trials from start Arm A and five 


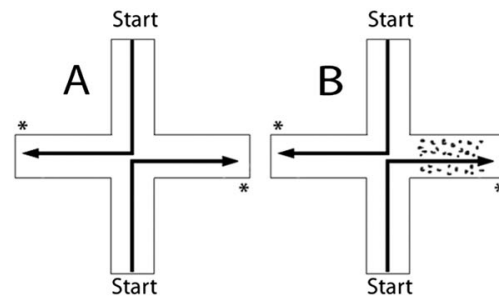

Fig. 2 Crayfish were tested in four different maze configurations and began trials from both start arms. Arrows indicate the correct path from each start arm and asterisks indicate the location of open exit doors. a

from start Arm B on each training day. Each crayfish was randomly assigned to exit left or right and, depending on the start arm, the open exit was located in either Arm C or Arm D. (2) Response learning with inconsistent place cues (response relevant). Here the maze contained place cues in either Arm $\mathrm{C}$ or Arm D, and the cues remained in the same arm for the duration of a particular animal's training. As in Group 1, crayfish were randomly assigned to always exit left or right, and they began five trials from start Arm A and five from Arm B. In this situation, only turn direction was relevant for accurate maze navigation, and crayfish had to rely on response learning in the presence of place cues that were inconsistent, being situated to the left or the right depending on the start location. (3) Place learning with inconsistent response cues (place relevant). For this group, the maze also contained place cues in $\mathrm{C}$ or $\mathrm{D}$, which remained in the same arm for the duration of an animal's training, and each animal began five trials from start Arm A and five from Arm B. Crayfish were randomly assigned to exit over the rough floor/toward the black stripes or over the smooth floor/toward the plain white panel, and the accurate place occurred to the left or right depending on the start arm. Here, only the external visual/tactile cues were relevant for maze navigation, and crayfish had to rely on the place cues in the presence of response information that was inconsistent. (4) Learning with redundant cues (both cues relevant). Crayfish in this group were assigned to exit left or right and always toward the same external cues. They began five trials from Arm A and five from Arm B, and the external cues were moved between Arms C and D. Because the cues moved, they no longer defined an absolute place in space, but in the featureless box they allowed the maze to appear identical to the animal regardless of start location (e.g., the rough floor/black strips always occurred in the right arm as the animal approached the maze center). Thus, crayfish could use response cues, external cues, or both combined to remember the exit location.

In Experiment $2(N=16)$, a subset of successful learners experienced five additional reversal trials on Days 8-12. Animals were considered successful learners if they achieved a mean score of $70 \%$ or higher for correct turns on the last 3 days of training. The subset consisted of animals that achieved the required mean score for correct turns and also continued to enter the exit compartment and the holding tank on every trial without extra turns or repeated delays lasting longer than 5 minutes. For reversal trials in the response-only and responserelevant conditions, the direction of the correct arm was switched (i.e., an animal trained to exit right had to exit left). In the both-cues-relevant condition, both the correct arm and the external cues were switched (i.e., an animal trained to exit right toward the black stripes and rough floor had to exit left toward a white wall and smooth floor). In pilot experiments, crayfish received up to 8 days of reversal training, but animals that had not learned the reversal by 5 days did not do so with extra training. This was mainly because animals that did not learn early in the reversal trials appeared to lose motivation and became less likely to enter exit compartments and leave the maze.

\section{Statistical analyses}

All statistical analyses were performed using SPSS with statistical significance set at $\alpha=.05$. In Experiment 1, we used a mixed ANOVA with one between-groups factor and one within-subjects factor to investigate the effects of training protocol across training days. In this test, training protocol was the between-groups factor with four levels, and training day was the within-subjects factor with seven levels (Days 1-7); the dependent variable was performance measured as the percentage of correct turns on each day. Where sphericity differed significantly (Mauchly's test of sphericity: $p<.05$ ), we report degrees of freedom and significance values based on the Greenhouse-Geisser estimate. Significant results were followed by post hoc Tukey tests to determine which groups differed significantly from each other in overall performance across all days. To further examine performance, we conducted followup tests of simple effects, focusing on the change in performance in each learning group across the training days. Specifically, we compared performance on Day 1, when animals experienced the initial set of maze trials with Days 2-7; we expected animals to display increased turn accuracy over the days of training and hence used one-tailed paired $t$ tests and the Bonferroni correction for multiple comparisons. In Experiment 2, a subset of animals that met the learning criteria defined above participated in reversal trials. Included in this 
subset were animals from three training protocol groups: response only, response relevant, and both cues relevant. We again used a mixed ANOVA with one between-groups factor (training protocol with three levels), one within-subjects factor (training day with five levels, Days 8-12), and one dependent variable (percentage of correct turns). We followed this analysis with a post hoc Tukey test to determine which groups differed significantly from each other in overall performance, and also examined performance in each group on each training day. First, to discover if turn accuracy was significantly affected by the reversal of cues, we used paired $t$ tests to compare percentage of correct turns on Day 7 with percentage of correct turns on Day 8, when the new task was first presented. To discover if turn accuracy improved over the days of reversal training, we used paired $t$ tests to compare performance on Days 9-12 with Day 8. We expected animals to display decreased accuracy on Day 8 compared with Day 7 and to improve performance over the 5 days of reversal training. Hence, we used one-tailed tests with alpha levels adjusted with the Bonferroni correction for multiple comparisons.

\section{Results}

A total of 56 crayfish ( $n=14$ /group) completed at least 7 days of training. The performance of animals in each of the four training groups across the 7 days is shown in Fig. 3. A mixed ANOVA found a main effect of learning group, $F(3,52)=$ $5.57, p=.002, \eta_{\mathrm{p}}{ }^{2}=.243$, a main effect of training day, $F(4.85,252)=8.39, p<.001, \eta_{\mathrm{p}}{ }^{2}=.139$, and a significant interaction between the two variables, $F(14.55,252)=3.061$, $p<.001, \eta_{\mathrm{p}}{ }^{2}=.150$. Post hoc Tukey tests revealed significant differences in overall performance between the response-only group and the place-relevant group $(p=.018)$, and between the both-cues-relevant group and the place-relevant group ( $p=$ .002 ), with the place-relevant group displaying less accurate performance in both comparisons. While mean accuracy in the response-relevant group showed a consistent tendency to be lower than both the response-only and both-cues-relevant groups, differences were not significant.

Our examination of performance in each learning group on each training day found that improvements in turn accuracy were significant in the response-only and both-cues-relevant groups, but not in the response-relevant and place-relevant groups. Specifically, in the response-only group, paired $t$ tests comparing the correct turns on Day 1 with subsequent testing days found that the difference was significant for Day $3, t(13)$ $=-3.58, p=.009, d=.96$; Day 4, $t(13)=-4.08, p=.003, d=$ 1.14; Day 5, $t(13)=-4.84, p<.001, d=1.42$; Day $6, t(13)=$ $-4.32, p=.003, d=1.24$; and Day $7, t(13)=-4.95, p<.001, d$ $=1.36$; but not Day 2, $t(13)=-2.35, p=.105, d=.64$. A total of nine out of 14 individuals $(64 \%)$ reached our learning criterion, and these animals achieved a mean score of $80 \%$ for correct turns on the last 3 days of training. Together, these data indicate that most crayfish were capable of remembering the maze exit based on egocentric cues only. In the tesponserelevant condition, differences in turn accuracy were insignificant between Day 1 and all subsequent Days: Day 2, $t(13)=$ $0, p=1.00, d=0$; Day 3, $t(13)=-1.12, p=.85, d=.30$; Day 4, $t(13)=-.61, p=1.00, d=.16$; Day $5 t(13)=-.57, p=1.00, d$ $=.16$; Day $6, t(13)=-.21, p=1.00, d=.06$; and Day $7, t(13)=$ $-1.75, p=.31, d=.47$. These results reflected the inconsistent performance of animals in this condition and suggests that the shifting place cues were distracting for most. However, six out of 14 individual crayfish (43\%) reached the learning criterion (mean score of $75 \%$ on the last 3 days) indicating that they were able to disregard the place cues and use egocentric information alone to orient. In the place-relevant condition, differences in turn accuracy were also insignificant between Day 1 and all subsequent days: Day $2, t(13)=1.99, p=.20, d=.54$; Day 3, $t(13)=1.23, p=.72, d=.53$; Day $4, t(3)=1.53, p=$ $.45, d=.41$; Day $5, t(13)=2.55, p=.07, d=.68$; Day $6, t(13)$ $=1.00, p=1.00, d=.27$; and Day $7, t(13)=.46, p=1.00, d=$ .12 . In this group, most animals performed inconsistently, and only one out of 14 (7\%) crayfish reached the learning criterion, achieving the minimum mean score of $70 \%$ correct on the last three training days. Finally, in the both-cues-relevant condition, paired $t$ tests found significant differences between performance on Day 1 and Day $5, t(13)=-3.42, p=.015, d$ $=.98$, and Day $7, t(13)=-4.34, p=.003, d=1.25$, but not on Day 2, $t(13)=-.64, p=1.00, d=.18$; Day 3, $t(13)=.28, p=$ $1.00, d=.08$; Day $4, t(13)=-2.02, p=.19, d=.56$; or Day 6 , $t(13)=-2.32, p=.11, d=.76$. Compared with the responseonly condition, animals in other groups performed more accurately on the first day, which may contribute to the lack of significant difference between Day 1 and other days despite relatively accurate performances in the both-cues-relevant condition. Examination of individuals in this group indicates that almost all animals were successful in learning the task: 13 out of 14 crayfish $(93 \%)$ reached the learning criterion with mean score of $80 \%$ in the last 3 days.

In Experiment 2, a total of 16 crayfish completed 5 days of reversal training, and data from these animals are shown in Fig. 4. Place relevant is not included because no animals in this group achieved the criteria for reversal training. A twoway mixed-model ANOVA was used to examine learning across learning groups and 5 days of reversal training and found a main effect of learning group, $F(2,13)=4.48, p<$ $.033, \eta_{\mathrm{p}}{ }^{2}=.41$, a main effect of training day, $F(4,52)=19.11$, $p<.001, \eta_{\mathrm{p}}{ }^{2}=.60$, but a nonsignificant interaction between the two variables, $F(8,52)=.95, p=.49, \eta_{\mathrm{p}}{ }^{2}=.13$. Post hoc Tukey tests revealed a significant difference between the response-only group and the both-cues-relevant group ( $p=$ .049), with the response-only group displaying less accurate performance. In each of the learning groups, animals displayed a significant decrease in accuracy on the first 


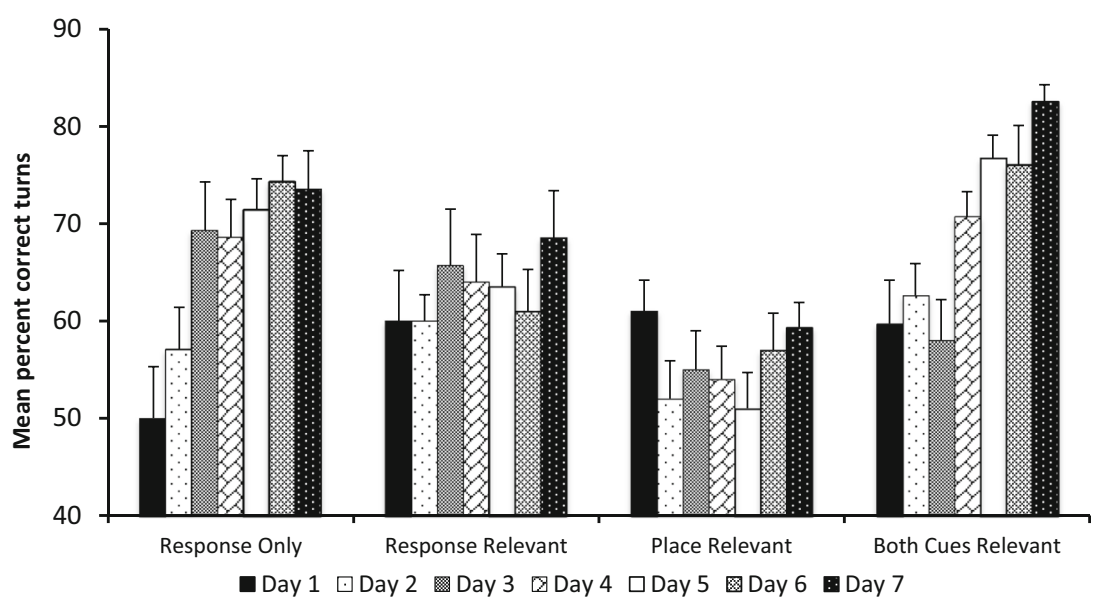

Fig. 3 Bars indicate the mean percentage correct turns $(+S E M)$ on each day of training for all animals in the four different training protocols $(N=14$ per training protocol). See text for statistically significant differences
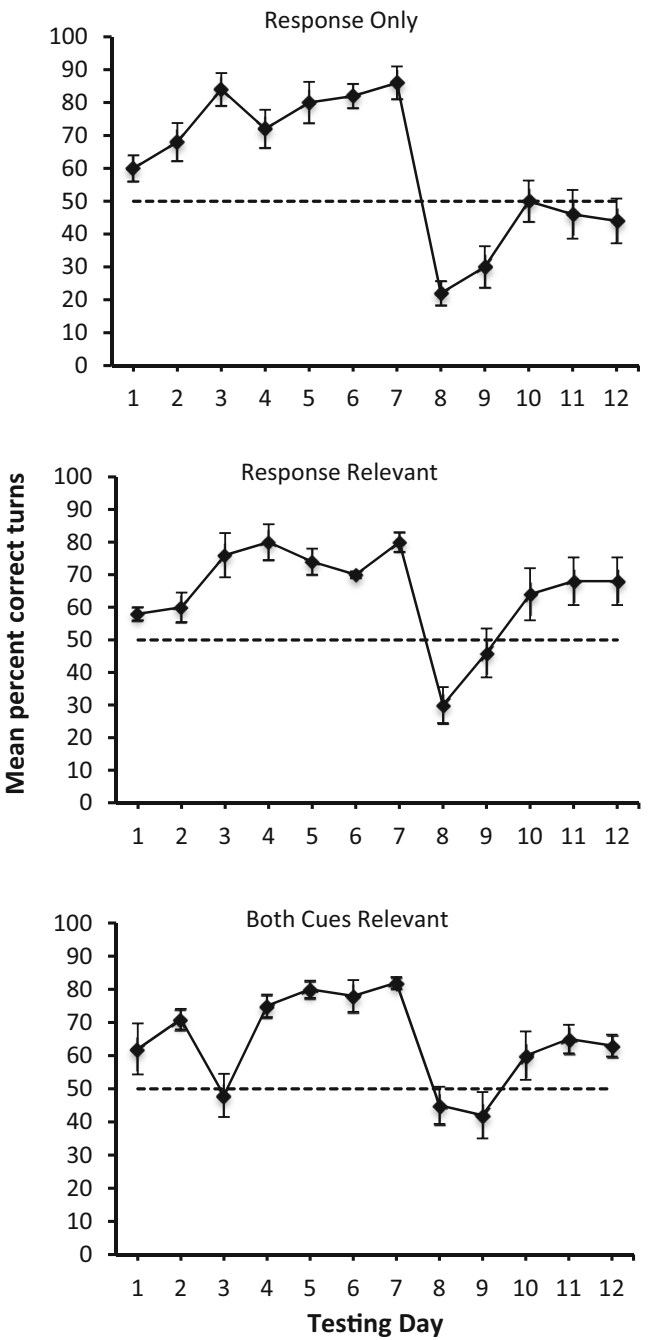

Fig. 4 Mean performance of crayfish which participated in reversal trials in the response-only $(n=5)$, response-relevant $(n=5)$, and both-cuesrelevant $(n=6)$ protocols. The response and/or place cues that led to an open exit door were reversed on testing Day 8 . See text for statistically significant differences reversal day (Day 8) compared with Day 7: response only, $t(4)$ $=8.55, p=.001, d=2.41$; response relevant, $t(4)=5.98, p=$ $.004, d=4.72$; both cues relevant, $t(5)=5.50, p=.003, d=$ 3.40. These data indicate that crayfish had learned the initial task and repeatedly attempted to turn in the previously rewarded direction on the first reversal day. Pairwise comparisons of performance on Day 8 with subsequent days revealed significant improvements in performance in the response-only group for Day 10, $t(4)=-4.80, p=.018, d=2.31$, but not Day $9, t(4)=-2.14, p=.20, d=1.31$; Day $11, t(4)=-2.95, p=.08$, $d=1.45$; or Day $12, t(4)=-2.56, p=.13, d=1.62$. In the response-relevant group, significant differences occurred for Day $10, t(4)=-3.67, p=.042, d=.83$, and Day $11, t(4)=$ $-3.73, p=.040, d=1.41$, but not for Day $9, t(4)=-1.73, p=$ $.32, d=.81$, or Day $12, t(4)=-3.413, p=.05, d=2.71$. Finally, for the both-cues-relevant group, significant differences occurred for Day 11, $t(5)=-3.87, p=.024, d=1.62$, and Day 12, $t(5)=-3.84, p=.024, d=1.72$, but not Day 9 , $t(5)=.42, p=1.00, d=.17$, or Day $10, t(5)=-2.24, p=.15, d$ $=.97$. These results suggest that, overall, animals were able to improve performance compared with the first day of reversal training, and some individual animals showed consistent improvement, supporting the idea that crayfish can learn a single reversal task (see Fig. 5). However, other animals, especially in the response-only group, remained at or below $50 \%$ accuracy on the final testing days, and none achieved the level of accuracy they had displayed during the initial training trials.

\section{Discussion}

Our results extend previous findings on spatial learning in $O$. rusticus and contribute new information on strategy use by this species. First, we manipulated training protocols such that the maze task required the use of response cues, external cues, or allowed simultaneous use of both cue types. We found that 


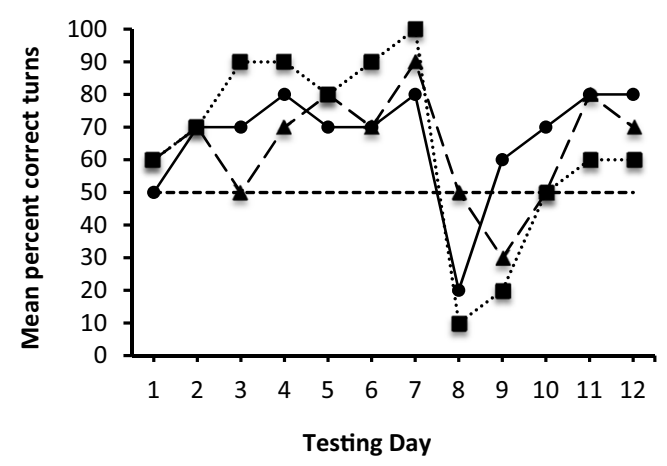

Fig. 5 Representative data from three individual crayfish that experienced reversal of cues on testing Day 8 after training in the response-only (dotted line, square markers), response-relevant (solid line, round markers), and both-cues-relevant (dashed line, triangle markers) protocols

crayfish could successfully learn a spatial task using response cues alone, but they were markedly less successful at learning the task using place cues when required to ignore inconsistent response information. Second, we hypothesized that performance would be enhanced if multiple cues were presented in a redundant format, and found that more animals successfully learned the task under these conditions compared with response learning formats. Third, we hypothesized that place learning, which could occur under two treatment conditions - place relevant and both cues relevant — would be associated with greater behavioral flexibility compared with response learning. We measured behavioral flexibility using a single reversal task and found that crayfish were able to learn the reversal when trained using response cues in the responseonly and response-relevant conditions, and using response and/or place cues in the both-cues-relevant condition. However, not all individuals learned the single reversal, and the presence of place cues did not consistently improve performance. Also, crayfish in the place-relevant group did not learn the initial task successfully and could not be tested in the reversal task. Hence, we did not find unequivocal support for the hypothesis that place learning allowed for greater behavioral flexibility than response learning.

Previous studies using probe (cue-conflict) trials after maze training found that crayfish oriented using place learning as frequently as response learning (Tierney \& Andrews, 2013; Tierney \& Lee, 2011). Hence, in the present study it was surprising to discover that crayfish learned much more successfully when response cues were relevant, with or without irrelevant place cues, compared with the condition where place cues were relevant and response cues were irrelevant. However, cue-conflict tests allow animals to choose freely between strategies when learning and also "guess" the correct (or incorrect) direction when confronted with a single forced choice between response and place information. A task that requires animals to consistently respond to a single cue type that is sometimes paired and sometimes mismatched with another cue poses a more difficult problem given that most animals use both internally and externally generated signals simultaneously (Wehner, Hoinville, Cruse, \& Cheng, 2016; White et al., 2013). However, with appropriate training, rodents readily learn to use only place or response information (Gibson \& Shettleworth, 2005), and fish are also capable of learning in this manner (Salas et al., 1996). Among invertebrates, desert ants learned to ignore path integration information in favor of landmark guidance when the landmark alone correctly marked the shifting location of a feeder (Wehner, 1970; Wehner et al., 2016). Thus, in some species, learning is among the factors that can change the weighted value (i.e., the importance) assigned to particular cues as animals navigate.

The weighted value of cues may also reflect ecological conditions and the evolution of spatial strategies, creating stable perceptual and motor biases. For example, Bruck, Allen, Brass, Horn, and Campbell (2017) used a maze task to compare two closely related mouse species, one of which burrows while the other lives above ground. When all external cues were unavailable and mice had to rely only on egocentric navigation, the burrowing species performed better than the surface species, suggesting that egocentric navigation is more heavily weighted in the former species, presumably as an adaptation to life underground. The ability of crayfish in our study to disregard external cues, but not egocentric information, suggests that the weighted value of the latter may be inherently greater in this species. Although $O$. rusticus is responsive to visual stimuli, it is possible that vision is less salient during navigation since this species is typically most active at night. Tactile cues would presumably be available in the dark, but may not offer as much information or be as easily remembered as a single turn direction. However, another nocturnal aquatic species, the chambered nautilus, displayed the opposite bias, orienting poorly when required to use egocentric information and much more accurately when visual cues were present (Crook \& Basil, 2012). Clearly, additional species must be studied to gain a better understanding of the selective pressures that shape the salience of egocentric and allocentric cues.

Although animals in the place-relevant group performed poorly, results from the response-relevant and both-cuesrelevant conditions suggest that crayfish were aware of the external cues. In the response-relevant group, there was no significant overall improvement in turn accuracy across the 7 days of testing, suggesting that crayfish noticed the "wrong" location of the place cues when started from opposite start arms, and most were disoriented by this. In both placerelevant and response-relevant groups, cue competition may have affected task performance since animals had to ignore irrelevant cues as well as remember relevant ones. However, six animals in the response-relevant group learned the task successfully, supporting the idea that certain individuals may 
especially be predisposed for response learning (Tierney \& Andrews, 2013). Interestingly, in the both-cues-relevant group, 93\% reached the learning criterion for the last 3 days of training compared with $64 \%$ in the response-only group. While additional data are needed to increase sample sizes, these findings offer preliminary support for the "additivity of cues" idea and are consistent with the hypothesis that redundant cues enhance spatial performance (Shettleworth, 2005). For animals that learned successfully, however, turn accuracy did not differ between response-only and both-cues-relevant groups. Also, the presence of multiple cues did not significantly enhance the speed of learning since both-cues-relevant animals did not improve more rapidly than response-only animals. Thus, while our protocol does not rule out simultaneous use of strategies, the availability of redundant external cues may have served primarily to allow learning in animals predisposed to use either place or response cues. This idea is consistent with previous findings that some individual crayfish preferentially orient using a place rather than a response strategy (Tierney \& Andrews, 2013).

An important difference between the present study and previous studies of crayfish orientation is that our apparatus eliminated extramaze visual information. Many previous studies have shown that animals make use of extramaze features (e.g., Rodriguez et al., 1994; White et al., 2013) or distant landmarks and panoramas in nature (M. Collett, Chittka, \& Collett, 2013; Wehner et al., 2016). Use of extramaze visual information has yet to be investigated in crayfish, but we note that animals in the present study were generally less successful in learning the task than when the maze was not contained within a featureless box (Tierney \& Andrews, 2013). Procedures used in these experiments also differed in that crayfish were allowed only a single entry into a maze arm. This eliminated the tendency of crayfish to wander and establish circuitous path habits (Tierney \& Lee, 2011), but did not appear to serve as an effective deterrent since many animals entered the wrong compartment repeatedly during training. Also, confinement may have made the procedure more stressful for the animals, which may have affected motivation or learning ability. Interestingly, stress and stress coping style may also affect spatial strategy choice in animals. For example, carp classified as bold and risk tolerant solved a spatial task with a fixed sequence of movements, whereas more cautious, risk-aversive animals used a place cue (Mesquita, Borcato, \& Huntingford, 2015). It is not known if stress coping style affects strategy choice in crayfish, but it is worth noting that the present maze task tends to favor bold animals.

Motivation and the contribution of motivation to task performance is also an important consideration in any learning experiment. In the present study, most animals immediately consumed the small amount of food available in the holding tank, suggesting that it served as an effective reward. However, it was not compelling reinforcement for all crayfish, because a significant number did not enter the holding tank and were eliminated in the course of training. Importantly, these animals did not fail to learn; rather, their learning could not be accurately assessed, because they repeatedly failed to perform the task of exiting the maze. Among animals that did complete the training, differences in motivation may have contributed to individual variability in behavior. For example, after choosing the correct exit compartment, most animals exited quickly, but some lingered on the stairs for several minutes. Occasionally, animals developed a habit of walking up to, but not into, the goal compartments, allowing them to walk back through the maze. A few especially large animals succeeded in climbing over the maze walls and, on subsequent trials, they persistently climbed in the exact location of the previous escape. Thus, idiosyncratic behavior produced many "results" that demonstrated precise memory of the maze environment and past experience, but did not contribute useful data on response and place learning. Our observations suggest that the most consistent behavior displayed by $O$. rusticus was exploration and attempts to escape from the maze, behavior that waned in some animals after they experienced the holding tank, another small, confined space. Hence, manipulations such as providing a much larger holding tank or escape to a home environment might be useful in future maze learning experiments.

Reversal learning indicates the ability to change behavior when environmental circumstances change and is commonly used as a measure of cognitive flexibility in animals. Our experiments demonstrated that some crayfish learned a single reversal in the context of response learning only or with place cues present. Previous studies reported that reversal learning was much better in place learners compared to response learners (Kleinknecht et al., 2012; Oliveira, Bueno, Pomarico, \& Gugliano, 1997), and the performance of response-only animals is consistent with this finding. Compared with the both-cues-relevant group, animals in the response-only group displayed significantly less accurate performance over the reversal training days. However, there was no significant difference between animals in the responserelevant and the both-cues-relevant groups, though consistent external cues were available only in the latter. Some animals in the both-cues-relevant condition may have used both response and external cues and hence may have perceived an especially complex reversal task. These animals would have had to respond to changes in both turn direction and external cues, whereas the obligatory response learners had to pay attention to turn direction only. Ideally, the use of place cues would be isolated from response learning, but the natural behavior of animals did not allow this comparison under our research conditions. Also, relatively few crayfish met the criteria for reversal training, and hence definitive conclusions regarding spatial and reversal learning await additional experimentation. 
Future studies in this area would be especially interesting. Relatively little is known about learning processes and cognitive flexibility in crayfish and other crustaceans. In addition, relatively little is known about coping style or, more generally, animal personality in crustaceans, despite high interest in this topic in other species (Roche, Careau, \& Binning, 2016; Sih \& Del Giudice, 2012). The intersection of learning and stress coping style yields testable hypotheses and promises to be an active area of research. For example, reactive animals are hypothesized to display greater behavioral flexibility than proactive animals, an idea that has been supported by better reversal learning in reactive animals of some species (e.g., Guenther, Brust, Dersen, \& Trillmich, 2014; Brust, Wuerz, \& Kruger, 2013), though not all (Bensky, Paitz, Pereira, \& Bell, 2017). Additional studies of learning and personality in crayfish or other crustaceans could yield discoveries in both areas, contributing needed taxonomic diversity to topics of wide interest to animal behaviorists.

Author note We thank the Colgate Research Council for contributing funding for this project, J. Wallace and I. Mackillop for constructive comments on the manuscript, and C. Baker and A. Schoonmaker for assistance with animal care.

\section{References}

Alves, C., Chichery, R., Boal, J., \& Dickel, L. (2007). Orientation in the cuttlefish Sepia officinalis: Response versus place learning. Animal Cognition, 10, 29-36.

Bensky, M. K., Paitz, R., Pereira, L., \& Bell, A. M. (2017). Testing the predictions of coping styles theory in threespined sticklebacks. Behavioural Processes, 136, 1-10. https://doi.org/10.1016/j. beproc.2016.12.011

Bhimani, R., \& Huber, R. (2016). Operant avoidance learning in crayfish, Orconectes rusticus: Computational ethology and the development of an automated learning paradigm. Learning \& Behavior, 44, 239 249.

Bruck, J. N., Allen, N. A., Brass, K. E., Horn, B. A., \& Campbell, P. (2017). Species differences in egocentric navigation: The effect of burrowing ecology on a spatial cognitive trait in mice. Animal Behaviour, 127, 67-73. https://doi.org/10.1016/j.anbehav.2017.02. 023

Brust, V., Wuerz, Y., \& Kruger, O. (2013). Behavioural flexibility and personality in zebra finches. Ethology, 119, 559-569.

Cannicci, S., Barelli, C., \& Vannini, M. (2000). Homing in the swimming crab Thalamita crenata: A mechanism based on underwater landmark memory. Animal Behaviour, 60, 203-210.

Capretta, P. J., \& Rea, R. (1967). Discrimination reversal learning in the crayfish. Animal Behaviour, 15, 6-7.

Collett, M., Chittka, L., \& Collett, T. S. (2013). Spatial memory in insect navigation. Current Biology, 23, R789-R800. https://doi.org/10. 1016/j.cub.2013.07.020

Collett, T., Graham, P., \& Durier, V. (2003). Route learning by insects. Current Opinion In Neurobiology, 13(6), 718-725.

Costanzo, D. J., \& Cox, W. G. (1971). Habit reversal improvement in crayfish. Journal of Biological Psychology, 13, 11-12.

Cronin, T. W., \& Goldsmith, T. H. (1982). Photosensitivity spectrum of crayfish rhodopsin measured using fluorescence of metarhodopsin. Journal of General Physiology, 79, 313-332.
Crook, R. J., \& Basil, J. A. (2012). Flexible spatial orientation in navigational strategies in chambered nautilus. Ethology, 119, 77-85.

Davis, K. M., \& Huber, R. (2007). Activity patterns, behavioural repertoires, and agonistic interactions of crayfish: A non-manipulative field study. Behaviour, 144, 229-247.

Drozdz, J. K., Viscek, J., Brudzynski, S. M., \& Mercier, A. J. (2006). Behavioral responses of crayfish to a reflective environment. Journal of Crustacean Biology, 26, 463-473.

Gibson, B., \& Shettleworth, S. (2005). Place versus response learning revisited: Tests of blocking on the radial maze. Behavioral Neuroscience, 119(2), 567-586.

Guenther, A., Brust, V., Dersen, M., \& Trillmich, F. (2014). Learning and personality types are related in cavies (Cavia aperea). Journal of Comparative Psychology, 128, 74-81. https://doi.org/10.1037/ a0033678

Kamran, M., \& Moore, P. A. (2015). comparative homing behaviors in two species of crayfish, Fallicambarus fodiens and Orconectes rusticus. Ethology, 121, 775-784. https://doi.org/10.1111/eth.12392

Kleinknecht, K. R., Bedenk, B. T., Kaltwasser, S. F., Grünecker, B., Yen, Y., Czisch, M., \& Wotjak, C. T. (2012). Hippocampus-dependent place learning enables spatial flexibility in C57BL6/N mice. Frontiers in Behavioral Neuroscience, 687. https://doi.org/10. 3389/fnbeh.2012.00087

McMahon, A., Patullo, B., \& Macmillan, D. (2005). Exploration in a Tmaze by the crayfish Cherax destructor suggests bilateral comparison of antennal tactile information. The Biological Bulletin, 208(3), $183-188$.

Mesquita, F. O., Borcato, F. L., \& Huntingford, F. A. (2015). Cue-based and algorithmic learning in common carp: A possible link to stress coping style. Behavioural Processes, 115, 25-29. https://doi.org/10. 1016/j.beproc.2015.02.017

Odling-Smee, L., Boughman, J., \& Braithwaite, V. (2008). Sympatric species of threespine stickleback differ in their performance in a spatial learning task. Behavioral Ecology and Sociobiology, 62(12), 1935-1945.

Oliveira, M., Bueno, O., Pomarico, A., \& Gugliano, E. (1997). Strategies used by hippocampal- and caudate-putamen-lesioned rats in a learning task. Neurobiology of Learning and Memory, 68(1), 32-41.

Packard, M., \& McGaugh, J. (1996). Inactivation of hippocampus or caudate nucleus with lidocaine differentially affects expression of place and response learning. Neurobiology of Learning and Memory, 65(1), 65-72.

Poldrack, R. A., \& Packard, M. G. (2003). Competition among multiple memory systems: Converging evidence from animal and human brain studies. Neuropsychologia, 41(3), 245-251.

Restle, F. (1957). Discrimination of cues in mazes: A resolution of the "place-vs. response" question. Psychological Review, 64, 217-228.

Roche, D. G., Careau, V., \& Binning, S. A. (2016). Demystifying animal 'personality' (or not): Why individual variation matters to experimental biologists. The Journal of Experimental Biology, 219, 38323843. https://doi.org/10.1242/jeb.146712

Rodriguez, F., Duran, E., Vargas, J. P., Torres, B., \& Salas, C. (1994). Performance of goldfish trained in allocentric and egocentric maze procedures suggests the presence of a cognitive mapping system in fishes. Animal Learning \& Behavior, 22(4), 409-420. https://doi. org/10.3758/BF03209160

Salas, C., Rodríguez, F., Vargas, J., Durán, E., \& Torres, B. (1996). Spatial learning and memory deficits after telencephalic ablation in goldfish trained in place and turn maze procedures. Behavioral Neuroscience, 110(5), 965-980.

Scapini, F. (2006). Keynote papers on sandhopper orientation and navigation. Marine and Freshwater Behaviour and Physiology, 39, 7385.

Schmidt, B., Jacobson, T. K., \& Markus, E. (2009). Hippocampal and striatal dependent navigation: Sex differences are limited to acquisition. Hormones and Behavior, 56(2), 199-205. 
Shettleworth, S. J. (2005). Taking the best for learning. Behavioural Processes, 69(2), 147-149.

Sih, A., \& Del Giudice, M. (2012). Linking behavioural syndromes and cognition: A behavioural ecology perspective. Philosophical Transactions of the Royal Society of London, Series B, 367, 27622772. https://doi.org/10.1098/rstb.2012.0216

Sutherland, N. S., \& Mackintosh, N. J. (1971). Mechanisms of Animal Discrimination Learning. New York, NY: Academic Press.

Tierney, A., \& Andrews, K. (2013). Spatial behavior in male and female crayfish (Orconectes rusticus): Learning strategies and memory duration. Animal Cognition, 16(1), 23-34. https://doi.org/10.1007/ s10071-012-0547-1

Tierney, A., \& Lee, J. (2011). Spatial learning in a T-maze by the crayfish Orconectes rusticus. Journal of Comparative Psychology (Washington, D.C.: 1983), 125(1), 31-39. https://doi.org/10.1037/ a0020866
Wehner, R. (1970). Die Konkurrenz von Sonnenkompass- und Horizontmarken-Orientierung bei der Wüstenameise Cataglyphis bicolor (Hymenoptera, Formicidae). Verhandlung Deutschen Zool Gesellschaft, 64, 238-242.

Wehner, R., Hoinville, T., Cruse, H., \& Cheng, K. (2016). Steering intermediate courses: Desert ants combine information from various navigational routines. Journal of Comparative Physiology, A, 202, 459472. https://doi.org/10.1007/s00359-016-1094-z

White, N., Packard, M., \& McDonald, R. (2013). Dissociation of memory systems: The story unfolds. Behavioral Neuroscience, 127(6), 813834. https://doi.org/10.1037/a0034859

Zeil, J. (1998). Homing in fiddler crabs (Uca lactea annulipes and Uca vomeris: Ocypodidae). Journal of Comparative Physiology A, 183, 367-377. 